Cultures \& Conflits

42 | été 2001

Le crime organisé en Russie : nouvelles approches

\title{
Entreprise, protection et violence en Russie à la fin des années 1990
}

\section{Vadim Radaev}

\section{(2) OpenEdition \\ 1 Journals}

\section{Édition électronique}

URL : http://journals.openedition.org/conflits/562

DOI : $10.4000 /$ conflits.562

ISSN : $1777-5345$

Éditeur :

CCLS - Centre d'études sur les conflits lilberté et sécurité, L'Harmattan

Édition imprimée

Date de publication : 1 juin 2001

ISBN : 2-7475-1117-0

ISSN : 1157-996X

\section{Référence électronique}

Vadim Radaev, «Entreprise, protection et violence en Russie à la fin des années 1990 », Cultures \& Conflits [En ligne], 42 I été 2001, mis en ligne le 20 mars 2006, consulté le 30 mars 2021. URL : http:// journals.openedition.org/conflits/562 ; DOI : https://doi.org/10.4000/conflits.562

Ce document a été généré automatiquement le 30 mars 2021.

Creative Commons License 


\title{
Entreprise, protection et violence en Russie à la fin des années 1990
}

\author{
Vadim Radaev
}

Selon une opinion courante, les entrepreneurs russes, évoluant dans un marché «sauvage ", ne partageraient actuellement ni règle ni convention ${ }^{1}$. Cette analyse est simpliste car les marchés émergents, tout comme ceux qui sont bien établis, ne sauraient se passer, au niveau des agents économiques, de confiance et d'engagements réciproques. Il est cependant vrai que l'action économique peut s'enraciner dans divers ethos et institutions. Trois grandes théories éthiques - égoïste, utilitaire, déontologique - indiquent à cet égard des distinctions commodes quant aux motivations des agents économiques. Quand ils s'inscrivent dans la logique de l'égoïsme, les agents poursuivent leurs intérêts économiques immédiats, tels qu'ils les perçoivent, sans référence aux codes moraux (c'est-à-dire aux règles de conduite qui reflètent les normes des affaires et de la vie civile) et aux conséquences futures de leurs actions. Pour autant qu'ils respectent les normes, c'est en raison des sanctions externes attachées à leur transgression. L'action utilitaire vise la maximisation du bien-être. Elle inscrit le calcul des intérêts, fussent-ils strictement égoïstes, dans une dimension temporelle. De ce fait, elle se soucie de la réputation professionnelle comme d'un «capital » organisationnel. Les agents agissent en conformité avec les normes parce qu'ils considèrent que, sur le long terme, l'honnêteté est payante. Dans l'action déontologique, on suppose que l'évaluation éthique est indépendante des intérêts économiques. De ce fait, les codes moraux ne reposent pas exclusivement sur des sanctions externes. Les agents sont motivés par leurs engagements et leurs obligations qui, à leur tour, se nourrissent de coutumes et de valeurs. "L'honnêteté, en fait, est avant tout un choix moral (...) Nous respectons nos promesses parce que nous pensons que la droiture l'exige, et non parce que c'est commercialement avantageux " ${ }^{2}$. Nous partons ici du principe que ces trois schémas analytiques ont leur validité : de fait, le plus souvent, les trois modes d'action coexistent, avec des combinaisons diverses, dans les relations économiques. C'est dans les sociétés plus ou moins stables que les motivations déontologiques sont les mieux fondées. A l'inverse, dans un contexte de changement social rapide, quand les normes et coutumes établies de longue date sont 
érodées, le poids relatif de l'égoïsme s'accroît. Les agents économiques tendent alors à négliger les codes moraux et les conséquences de leurs actions. Les contraintes qui pèsent sur eux sont alors principalement les sanctions externes, $\mathrm{y}$ compris la menace de la coercition. La montée de l'égoïsme est incontestablement perceptible dans la Russie post-communiste contemporaine. Toutefois, il ne faut pas y voir une absence d'éthique des affaires, mais plutôt l'émergence de codes moraux spécifiques. A partir des données empiriques les plus récentes qui sont à notre disposition, cet article se penchera sur l'extension de la violence dans les affaires russes et sur les relations spécifiques qui se mettent en place dans le champ de la protection économique ${ }^{3}$. La violence dans les affaires économiques russes En utilisant à la fois des sources statistiques et des données d'entretien, nous présentons ci-après des éléments sur l'extension de la violence et sur les différentes tendances auxquelles elle donne lieu. L'extension des menaces et de la violence La persuasion informelle des "partenaires » au sens large (associés, clients, fournisseurs, débiteurs, créanciers, etc.), au moyen de menaces ou de violences ostentatoires, joue, de l'avis général, un rôle important dans l'activité entrepreneuriale en Russie aujourd'hui. D'après des sources médiatiques, qui rendent compte de données produites par la Chambre russe du Commerce et de l'Industrie, jusqu'à $70 \%$ des entreprises sont contraintes de verser des sommes importantes pour leur " protection " ${ }^{4}$. Nos données d'enquête conduisent à nuancer ce chiffre. $79 \%$ des entrepreneurs de notre échantillon expriment leur accord avec l'affirmation selon laquelle les menaces et la force sont utilisées aujourd'hui dans les affaires économiques russes. Pour $17 \%$ d'entre eux, c'est un phénomène fréquent ; pour $62 \%$, un phénomène occasionnel. L'expérience personnelle de telles exactions est toutefois plus rare. Moins de la moitié (42\%) des entrepreneurs interrogés y a été personnellement exposée, dont $3 \%$ souvent, et $39 \%$ occasionnellement (voir tableau 1). L'image médiatique d'une économie russe complètement " criminalisée » apparaît donc quelque peu exagérée. D’autres éléments émergent sur la localisation de la violence économique en Russie. La violence se concentre dans des secteurs à faible intensité capitalistique, où circulent des sommes importantes d'argent liquide, et dont les techniques de gestion sont relativement simples. Les secteurs "sophistiqués ", qui nécessitent de fortes compétences professionnelles, semblent être davantage préservés. Plus les processus de décision sont simples dans l'entreprise convoitée, plus les tentatives de pression ont des chances d'aboutir. La violence s'oriente par ailleurs de manière préférentielle vers les secteurs qui consacrent un budget important aux dépenses publicitaires et qui sont caractérisés par une forte visibilité. L'hypothèse d'une corrélation positive entre dépenses publicitaires et expérience personnelle de l'extorsion est confirmée par nos données. On constate enfin une certaine stabilisation des trajectoires (path dependence). Les entreprises qui ont opéré dans des marchés ou des secteurs plus ou moins criminalisés tendent à s'y spécialiser. Tableau 1. L'usage des menaces et de la force dans les affaires russes (selon l'opinion ou l'expérience des entrepreneurs interrogés ; pourcentages en ligne)

Fréquent Occasionnel Absent Dans les affaires russes en général 176221 Fréquent Occasionnel Absent Dans votre expérience personnelle 33958 Plus fréquent Aucun changement Moins fréquent Changement au cours des 2 ou 3 dernières années 145630 Impossible Difficile Possible Possibilité d'éviter la contrainte 83458

Les secteurs particulièrement touchés par la violence comprennent notamment le commerce de gros, le commerce de détail, la restauration collective, les services au consommateur, les services financiers. Les entreprises industrielles et scientifiques, 
tout comme les secteurs de la santé et de la culture, sont moins concernés ${ }^{5}$. La violence dans les relations contractuelles Le non-respect de contrats d'affaires fonde l'extension de la violence dans la Russie contemporaine. $92 \%$ des entrepreneurs reconnaissent que les contrats sont violés dans les affaires économiques russes ; $8 \%$ seulement prétendent ne pas connaître ces pratiques. Pour $49 \%$ des entrepreneurs, de telles violations sont courantes. Ces chiffres sont analogues à ceux de l'enquête de 1993 : aucune progression ne s'observe de ce point de vue. Dans leur activité quotidienne, les entrepreneurs disent être confrontés au non-respect de contrats : Fréquemment $32 \%$ Parfois $50 \%$ Jamais $18 \%$

Un comportement opportuniste serait ainsi courant dans le monde des affaires. Comment les entrepreneurs réagissent-ils habituellement à des tentatives de fraude ou à des manquements contractuels? Les stratégies seraient les suivantes : négociation / persuasion $55 \%$ recours à une cour d'arbitrage ${ }^{6} 24 \%$ recours à la force $11 \%$ rétorsion commerciale $3 \%$ aucune action $4 \%$ La violence est donc explicitement reconnue comme une modalité des relations commerciales. Certes, le recours à la force n'est cité que par une petite minorité. La majorité des entrepreneurs, à l'inverse, dit préférer la négociation. Celle-ci, toutefois, ne se limite pas à l'appel au respect de principes juridiques ou éthiques généraux. La menace de la force - par référence implicite à des agences de protection dotées de capacités coercitives - se situe au cœur des tactiques de négociation. Cette menace voilée est, de surcroît, jugée généralement efficace : elle facilite plutôt qu'elle n'entrave la coopération. Ainsi la violation habituelle des contrats favorise une éthique des affaires qui n'intègre ni valeurs généralement partagées, ni rentabilité attendue de l'honnêteté. De même, le droit n'y occupe qu'une place mineure. Cette éthique dérive des pratiques quotidiennes de lutte contre la violation systématique des contrats et donne naissance à une raison pratique dans laquelle la violence offre des ressources pour contrer l'opportunisme. Mais l'usage direct de la violence est, de l'avis général, à la fois coûteux et risqué. Celui qui y a recours peut à son tour devenir victime dans un proche avenir. Tout en étant structurée par la violence, la raison pratique des affaires amène, par ce biais, les agents économiques à une certaine prudence dans l'évaluation des conséquences de leurs actions, et donc à écarter l'usage effectif de la violence dans la mesure du possible. Le marché des services de protection est désormais bien développé en Russie. Il se répartit entre les types d'agences suivants: agences d'Etat; agences privées légales créées par d'anciens responsables de la sécurité d'Etat; agences privées légales créées par des criminels; agences illégales. La croissance du secteur de la protection privée date de la promulgation, le 11 mars 1992, de la loi fédérale "sur les activités privées d'investigation et de protection ». Après une vive augmentation du nombre d'agences en 1993-1994, le marché s'est stabilisé en 19967. Au début de 1999, environ 11.000 agences de sécurité étaient enregistrées en Russie et employaient quelques 160.000 salariés brevetés, détenant environ 71.000 armes déclarées ${ }^{8}$. Ces agences ne sont pas toutes actives, surtout depuis la crise qui a frappé ce secteur en 1998, à la suite de laquelle nombre d'agences furent fermées par les autorités. Elles n'en restent pas moins nombreuses : dans la seule ville de Moscou, il y aurait actuellement 871 entreprises de sécurité privée et environ 42.500 armes à feu déclarées en leur sein ${ }^{9}$. Les agences de protection ne se contentent plus d'imposer leurs services à leurs clients : l'époque du racket pur et simple est terminée. Au-delà de l'extorsion, la protection est un véritable commerce qui repose sur des ententes stratégiques entre partenaires. Les principaux services proposés par les agences sont la protection des biens et du personnel, 
l'information sur les activités de partenaires, le contrôle des transactions par la coercition et la mise à disposition de réseaux de relations (y compris avec l'Etat) afin de faciliter la résolution des difficultés commerciales et financières. Ainsi, au-delà de la protection de la vie et de la propriété, les agences de protection privées et semi-privées se consacrent activement au respect des contrats. Elles jouent un rôle influent - peu à peu devenu indispensable dans la vie des affaires - de tiers exerçant par délégation des fonctions importantes en matière de contrôle des transactions importantes (soprovojdenie sdelok). Les agences de sécurité (aussi bien légales que criminelles) assurent également des services particuliers, comme le recouvrement de créances auprès de débiteurs peu fiables ou franchement malhonnêtes. Une forte concurrence caractérise le marché des services de protection. Elle ne porte pas uniquement sur les prix et n'est pas propre au secteur privé. Les agences d'Etat cherchent à accroître leur part de marché - notamment le FSB (Service fédéral de sécurité), le MVD (Ministère de l'Intérieur) et la Police fiscale d'Etat - par le biais d'une commercialisation croissante de leur activité1 ${ }^{10}$. Elles vendent leurs services dans l'économie grise aussi bien que dans le secteur formel. A la faveur de cette concurrence, les agences d'Etat ont inévitablement affaire aux groupes criminels organisés. Selon les entrepreneurs interrogés, les deux types d'agences agissent de manière similaire. Il ne faut pas en conclure que, selon le cliché médiatique, l'Etat russe est « mafieux ». Il convient plutôt d'y voir un système flexible et à bien des égards fonctionnel de liens informels entre agences étatiques, para-étatiques et criminelles. Les principales fonctions de ce système sont la répartition des sphères d'influence et des clients solvables, l'obtention d'une rémunération complémentaire pour les agents administratifs et le contrôle des secteurs criminels. La modification de la répartition des marchés, qui se produit de temps en temps, se fonde sur les capacités coercitives relatives des agences de protection ainsi que, de manière croissante, sur leur capacité à établir des contacts informels avec les pouvoirs publics et les acteurs influents du secteur. Choix de partenariats et coûts de transaction Toute stratégie économique efficace passe désormais par de bonnes relations avec des agences de protection. Les entrepreneurs ont, du fait de la concurrence, une certaine liberté, aussi structurellement limitée soitelle, dans le choix de leurs partenaires. En revanche, ce choix crée un rapport de dépendance : tout changement ultérieur suppose l'appel à une agence concurrente plus puissante. Les enjeux de la sélection ressortent de nos données empiriques. Comment un entrepreneur qui a reçu des menaces réagit-il le plus souvent? Les réponses se distribuent de la façon suivante : en se débrouillant seul $34 \%$ en s'adressant à la police $13 \%$ en s'adressant à une agence de sécurité légale $8 \%$ en s'adressant à un groupe criminel $15 \%$ difficile à dire $30 \%$ Ce sont les petites entreprises qui ont le plus souvent affaire aux groupes criminels et semi-criminels. Leurs gérants s'adressent moins souvent à la police. En outre, les petits entrepreneurs sont moins nombreux à répondre de manière évasive, ce qui suggère que les problèmes de sécurité sont plus pressants pour eux que pour les dirigeants d'entreprises plus grandes.

Tableau 2. Réactions des entrepreneurs aux menaces, selon la taille de l'entreprise (pourcentages en colonne) Petites entreprises Entreprises grandes et moyennes En se débrouillant seul 3530 En s'adressant à la police 1219 En s'adressant à une agence de sécurité légale $104 \mathrm{En}$ s'adressant à un groupe criminel organisé 174 Difficile à dire 26 43

Le recours aux services des agences de protection est une source de coûts supplémentaires de transaction pour les entreprises: salaires des agents, paiements 
contractuels aux sociétés de sécurité, paiements en contrepartie des services additionnels associés à l'usage de la violence. Nous disposons de certaines données quantitatives sur les dépenses des entreprises en matière de protection. Plus de la moitié des entrepreneurs interrogés déclarent engager de tels coûts. Les deux tiers considèrent ces coûts comme insignifiants. Pour l'autre tiers, les coûts sont jugés significatifs, et leur montant croît (sans surprise) avec le degré d'exposition à la violence dans les relations d'affaires. Le prix de ces «services" peut être élevé. Par exemple, en matière de recouvrement de créances, les groupes criminels exigent en général la moitié de la somme recouvrée. Les commissions des agences légales iraient de 15 à $40 \%$ de l'encours de la créance ${ }^{11}$. Il arrive que les agences de sécurité proposent un ensemble de services récurrents contre une "prime d'assurance». Les agences légales facturent de tels services entre 100 et 600 USD par mois, selon la taille de l'entreprise et la gamme de services proposés ${ }^{12}$. Quand les agences de sécurité sont plus étroitement associées aux affaires de l'entreprise, les coûts peuvent être beaucoup plus importants, jusqu'à 10-15\% du chiffre d'affaires. Ils peuvent évidemment être encore bien supérieurs quand les agences de sécurité, au-delà de leurs services ordinaires, participent au capital de l'entreprise. Tendances récentes et routinisation de la violence Selon les données quantitatives disponibles, l'évaluation des tendances récentes peut conduire à un certain optimisme. $30 \%$ des entrepreneurs pensent que les tentatives d'usage de la force deviennent moins fréquentes. En même temps, la majorité d'entre eux (56\%) pensent que la situation en matière de violence dans les affaires est inchangée sur les deux ou trois dernières années (14 \% pensent que la situation s'est dégradée). L'optimisme porte plutôt sur la possibilité d'éradication du phénomène. $8 \%$ seulement des entrepreneurs refusent d'envisager une telle amélioration, alors que $58 \%$ d'entre eux la considèrent comme tout à fait possible (pour $34 \%$, la violence sera difficile à éviter entièrement ; voir tableau 1). Les données d'entretien se révèlent beaucoup plus positives quant à une éventuelle réduction de la place de la violence dans les affaires russes qu'au début des années 90. Plusieurs arguments étayent une telle appréciation. Tout d'abord, le crime organisé est attiré par les secteurs où le profit est rapide et aisé. Or, ces secteurs, en dehors des marchés "noirs" des stupéfiants ou des armes, sont désormais en voie de disparition. "L'époque du risque élevé, quand on pouvait faire beaucoup d'argent rapidement, est terminée. Désormais, il faut travailler dur au quotidien, sans engranger de superprofits. De ce fait, il n'y a plus d'extorsion » (dirigeant de société immobilière). Ensuite, le partage initial des zones d'influence entre agences légales et illégales de sécurité privée, ainsi qu'entre les différents groupes criminels, a été accompli, même si des conflits se produisent chaque fois que la définition de leurs frontières est remise en question. Les sanctions à l'encontre de ceux qui transgressent les frontières établies peuvent être extrêmement sévères, mais cela ne concerne pas la plus grande partie des entrepreneurs. "Toutes les zones d'influence ont été divisées aujourd'hui. Les 'krychi' [groupes de protection] sérieux n'ont plus rien à diviser" (dirigeant de société commerciale). Troisièmement, les groupes criminels organisés ont subi des changements importants. Ils se sont professionnalisés et ont commencé à s'enraciner dans des secteurs " gris » et "blancs ». Ils répondent ainsi à l'exigence de blanchir l'argent sale, tout en aspirant également à une image d'« hommes d'affaires honnêtes ». Ils doivent donc adopter au moins certaines des règles établies du monde des affaires, $\mathrm{y}$ compris lorsqu'elles sont en contradiction avec leur code criminel. L'opposition entre affaires et activités criminelles s'efface au profit d'une certaine interpénétration. Bien 
entendu, la décrue de la violence explicite ne signifie nullement la disparition de la criminalisation de l'économie, mais plutôt qu'elle se transforme, se routinise et devient un élément «normal » des relations économiques. Le racket plus ou moins " sauvage » a été remplacé par des formes plus stables et plus « civilisées » de contrôle du crime organisé sur les entreprises. Comment s'établissent de nouvelles formes d'ordre et de contrôle dans un tel contexte? Tout d'abord par un processus d'identification et de démarcation selon lequel les agences habilitées à exercer la violence dans certains marchés sont définies et légitimement fondées à exclure les nouveaux venus. Les rapports entre entrepreneurs et agences de protection se fondent sur une éthique spécifique fondée sur le respect de la force. C'est une sorte de droit coutumier qui s'appuie sur le recours éventuel à la violence. Ce qui assoit la légitimité des agences habilitées, c'est moins un code moral strict que l'effectivité de leur position de puissance. C'est la capacité de contrôler, dans la durée, telle zone d'influence qui fonde le droit exclusif d'y commercialiser des services de protection. Evolution des agences de protection étatiques Un certain nombre d'éléments suggèrent, au moins à titre de tendance générale, que les agences étatiques, avec leurs associés privés, ont réussi depuis quatre ou cinq ans à évincer les groupes criminels de plusieurs marchés importants. Cela résulte d'une évolution frappante des agences étatiques de protection russes au cours des années 90. Evolution quantitative, tout d'abord: le nombre de policiers («miliciens » ordinaires, à l'exclusion des éléments militaires rattachés à la police) est passé de 463.600 en 1992 (40,4 pour 10.000 habitants) à 601.100 en 1998 (63,8 pour 10.000 habitants). Simultanément, les agences étatiques ont également été renforcées du point de vue de leur encadrement, de leur équipement et de leur organisation. De l'avis général, les agences étatiques offrent un niveau de sécurité supérieur à un coût moindre. En effet, leurs options légales sont plus larges et leur structure de risque est avantageuse : elles sont en mesure de transférer à l'Etat une part importante de leurs coûts, alors que leurs bénéfices sont privatisables. S'il est vrai que la plupart des entrepreneurs seraient certainement ravis de jouir de la protection ( "krycha ») de la police - ou, mieux, du Service Fédéral de Sécurité -, leur liberté de choix n'en est pas moins structurellement limitée. De tels services exigent des capacités économiques suffisantes ou des relations personnelles avec les agences de protection, qui sont davantage à la disposition des grandes entreprises, publiques ou privées. Il en résulte une segmentation du marché de la protection selon le statut des entreprises. De manière circulaire, l'accès différentiel à la protection (étatique, privée ou criminelle) tend à pérenniser les différences de statut entre entreprises. "Ceux qui font des affaires illégales (vente sans licence de vodka et de bière, de véhicules, d'armes, ou trafic de drogue) attirent les groupes criminels. Les autres entrepreneurs cherchent à bénéficier de la protection de la police fiscale, du Service Fédéral de Sécurité, de la police, etc. Ces agences offrent des services de meilleure qualité à un coût raisonnable. Bien sûr, elles interviennent alors à titre informel » (dirigeant de société industrielle). L'entrepreneur face à la violence : une typologie Les deux facteurs qui nous semblent fondamentaux pour élaborer une typologie des entrepreneurs face à la violence sont la fréquence de la confrontation à la violence (avis général et expérience personnelle) et les dépenses effectuées par l'entreprise pour sa protection et les "services informels ». Trois grands groupes d'entrepreneurs peuvent être distingués :

Protégés $43 \%$ de l'échantillon Résistants $28 \%$ Vulnérables $29 \%$

Les entrepreneurs protégés sont confrontés à la force moins souvent que la moyenne. Seuls $43 \%$ d'entre eux considèrent que la violence est un facteur important dans les 
affaires russes, et $5 \%$ seulement y sont confrontés dans leur propre activité. Ils partagent également une évaluation plus optimiste à la fois des tendances récentes en la matière et de la possibilité pratique d'éviter la violence dans les relations d'affaires (voir tableau 3). Trois hypothèses peuvent être avancées pour expliquer les spécificités de ce groupe : soit les entreprises concernées sont faiblement visibles et échappent à l'attention des agences de protection, soit elles sont insuffisamment rentables pour les attirer, soit leur sécurité est assurée par un dispositif efficace de protection. L'analyse des données confirme plutôt la troisième hypothèse. Tout d'abord, les entreprises du premier groupe ne sont pas particulièrement discrètes : 35 \% d'entre elles engagent des dépenses publicitaires importantes. Ensuite, elles sont clairement concernées par la protection : les trois-quarts engagent des dépenses de sécurité, dont $22 \%$ à un niveau significatif. Le groupe se caractérise également par une part plus importante que la moyenne de coûts d'exploitation consacrés aux services informels. En conséquence, la relative absence de violence chez les entreprises de ce groupe résulte d'un niveau élevé de protection. Elles disposent de réseaux informels et de services internes de sécurité efficaces et appropriés. C'est pourquoi près de la moitié ( $47 \%)$ des entrepreneurs du premier groupe opte pour essayer de gérer par eux-mêmes d'hypothétiques menaces ou exactions violentes à leur encontre. Ils ne seraient qu'une petite minorité à s'adresser à des groupes criminels ( $12 \%)$ ou à la police $(7 \%)$. Sur la plupart des autres critères, ces entreprises sont proches de la moyenne, si ce n'est qu'elles sont légèrement plus orientées vers la production industrielle. La situation des entrepreneurs résistants est plus compliquée. Indéniablement confrontés à l'usage de la violence ( $88 \%$ d'entre eux considèrent que la violence est fréquente dans les affaires russes en général, et $37 \%$ se déclarent touchés par ce phénomène dans leur propre activité, mais de manière occasionnelle - voir tableau 3), ils consacrent peu de ressources à la protection. Ils évaluent de manière plutôt pessimiste les tendances dans ce domaine. Pour $11 \%$ d'entre eux, il est absolument impossible d'échapper à la violence dans les circonstances actuelles. Trois explications peuvent fonder ces constats: soit le groupe d'entrepreneurs considéré dispose de moyens financiers insuffisants pour assurer sa protection, soit il cherche à éviter tout contact avec les agences de protection, soit il développe sa propre résistance à l'extorsion en respectant les règles formelles de l'activité économique. Chacune des trois hypothèses est étayée par certains éléments empiriques, mais c'est la troisième qui semble la plus pertinente. Tout d'abord, aucune entreprise de ce groupe n'affecte à la protection et aux services informels des moyens importants, bien que la plupart aient des problèmes évidents de sécurité. Les entreprises du groupe 2 n'ont pas en interne de départements de sécurité puissants. Seuls $29 \%$ des entrepreneurs compteraient sur leurs propres capacités face aux menaces ou à l'usage de la force. Ils sont nombreux à ne pas savoir comment ils réagiraient (un tiers des personnes interrogées ne répondent pas à cette question). Ensuite, ces entreprises sont beaucoup moins nombreuses (22\%) à faire la publicité de leurs activités. Elles sont réticentes à s'ouvrir vers l'extérieur et à attirer l'attention d'envahisseurs potentiels. Enfin, elles peuvent élaborer une véritable stratégie de résistance, ce qu'attestent les données suivantes. Les entrepreneurs, en effet, trouvent généralement des méthodes formelles de résolution de leurs problèmes. Ils représentent les entrepreneurs les plus «légalistes» de l'échantillon, les moins impliqués dans des transactions informelles. Ils seraient plus nombreux à recourir à la police en cas de violence (19\%), et à l'arbitrage en cas de comportement déloyal (29\%). Le recours à la force ne concernerait que 3,5\% des membres du groupe. Moins 
impliquées dans l'économie informelle, ces entreprises seraient également moins attirantes pour les agences de protection. On trouve, dans ce groupe, une présence relativement forte d'entreprises, privatisées ou non, travaillant dans les secteurs de la science, de la santé et de la culture. Les entrepreneurs vulnérables constituent un troisième groupe, différent des autres car soumis à une forte pression de la violence. Ce sont les entreprises qui dépensent le plus en services de protection et autres services informels plus ou moins liés à la sécurité. Tous les entrepreneurs de ce groupe considèrent la violence comme caractéristique des affaires en Russie (pour $36 \%$, elle est fréquente) ; $95 \%$ d'entre eux sont directement concernés (dont $11 \%$ fréquemment). Ils sont les plus pessimistes en ce qui concerne les tendances actuelles et la possibilité d'échapper à la violence. Plus de la moitié d'entre eux (55\%) estiment que la libération des entreprises russes de l'emprise du crime organisé doit aujourd'hui représenter un objectif prioritaire pour le gouvernement. Sur tous ces points, ce groupe d'entrepreneurs se distingue par les indicateurs les plus élevés (tableau 3). L'existence de ce groupe peut être liée aux deux facteurs suivants: soit sa visibilité attire particulièrement les agences de protection, soit il est davantage impliqué dans les activités informelles, ce qui le rend potentiellement plus vulnérable à la violence. La première hypothèse est étayée par le fait que près de la moitié des entrepreneurs consacrent à la publicité des dépenses importantes. Ils attirent donc les agences de protection susceptibles d'offrir (ou d'imposer) leurs services. Leur vulnérabilité est accrue par une implication plus grande dans les activités informelles ou criminelles. Pour $23 \%$ d'entre eux, le recours aux groupes criminels serait la réponse prioritaire en cas d'exactions. Près d'un quart utiliserait directement la force pour contraindre un partenaire récalcitrant. Ce sont les membres de ce groupe qui sont les plus sceptiques quant au recours au droit. Ces données tendent à étayer la seconde hypothèse. Sans surprise, les entreprises de ce groupe relèvent pour la plupart du commerce de gros et de détail, de la restauration, et des services au sens large. Moscou y est fortement représenté ( $27 \%$ du groupe) : la capitale attire, en effet, à la fois les gros capitaux et ceux qui les convoitent.

Le poids relatif de l'égoïsme économique s'est accru dans la période post-communiste. Les agents poursuivent souvent leurs intérêts économiques immédiats, tels qu'ils les perçoivent, sans se soucier des codes moraux ou des conséquences de leurs actions. Dans ce sens, leurs actions ne sont influencées que par des contraintes extérieures, notamment coercitives. Cela ne signifie pas qu'il n'y ait aucune éthique des affaires, mais plutôt que les codes moraux applicables y prennent une forme spécifique. La Russie post-communiste est marquée par l'insécurité des relations économiques. Il est courant que les entrepreneurs subissent des menaces ou des exactions violentes. Ils sont contraints de faire appel aux services coûteux des agences de protection légales et /ou criminelles. Il en résulte des codes de comportement spécifiques, qui sont encadrés par la violence potentielle plutôt que par le droit ou par des considérations éthiques supérieures. La violation fréquente des contrats contraint les entrepreneurs à recourir à la violence pour protéger leurs actifs et leurs revenus. Bien que l'incidence de la violence et de la protection criminalisée semble avoir été exagérée par les médias, la force est incontestablement devenue une modalité "normale" des relations économiques en Russie. L'intensité de la violence dans les relations économiques horizontales (client - fournisseur, débiteur - créancier, etc.) décroît peu à peu. Moins que d'une décriminalisation, il s'agit d'une routinisation de la violence et de la stabilisation d'une nouvelle éthique fondée sur les capacités coercitives. Les agences de 
sécurité étatiques ont connu des changements considérables durant les cinq dernières années. Dans l'ensemble, leur position s'est renforcée. Mais c'est au prix d'une commercialisation de l'Etat et d'une interaction constante avec les agences semi-légales et criminelles. Nous évaluons à environ $15 \%$ la part d'entrepreneurs susceptibles de recourir à des formes criminelles d'activité. Toutefois, la proportion des entrepreneurs impliqués dans les segments "gris» de l'économie informelle, et mêlés au moins occasionnellement à des activités extra-légales, est beaucoup plus importante.

Tableau 3. Recours aux menaces et à la force par type d'entrepreneur (pourcentage en colonne, sauf pour le nombre d'entrepreneurs).

Protégés Résistants Vulnérables Nombre d'entrepreneurs 865658 Type d'entreprise Entreprises d'Etat privatisées 152114 Petites entreprises 817984 Le recours aux menaces et à la force

- S'observe dans les affaires russes en général 4388100

- dont fréquemment 01636

- Représente un phénomène auquel j’ai été personnellement confronté 53795

- dont fréquemment 1011

- S'observe plus fréquemment depuis deux ou trois ans 51620

- Est incontournable dans les affaires 7115

Comment réagissez-vous aux menaces ou à la force dans les relations d'affaires?

- en essayant de me débrouiller seul 472936

- en m'adressant à la police 71913

- en m'adressant aux agences de sécurité 12611

- en m'adressant aux groupes criminels $12 \quad 14 \quad 23$ Comment réagissez-vous aux comportements déloyaux de vos partenaires?

- en recourant à l'arbitrage 222914 - en recourant à la force 103.523 Est-ce que vous consacrez des dépenses significatives

- à la protection? 22043

- aux services informels ? 24030 - à la publicité ? 352247 Considérez-vous que les entrepreneurs doivent se libérer de l'emprise des groupes criminels? 323655 Annexe méthodologique ${ }^{13}$ Sources L'analyse se fonde sur des données recueillies dans le cadre de deux enquêtes réalisées en 1997-98 sur les dirigeants d'entreprises privées et les entrepreneurs (réunis sous le terme " entrepreneurs » dans ce qui suit). Ces enquêtes, spécifiquement conçues pour analyser le jeu de la violence et de la protection économiques, comprennent : un recueil de données standardisées (227 questionnaires auprès d'entrepreneurs de 21 régions de Russie); un recueil de données semistandardisées (96 entretiens approfondis avec des entrepreneurs). Les enquêtes ont été réalisées par l'auteur et par l'équipe de recherche du Centre d'Etude des Technologies Politiques à Moscou, sous la direction de I. Bounin. La recherche a bénéficié du soutien financier du Centre for International Private Enterprise (CIPE) aux Etats-Unis. Les résultats sont présentés sous forme quantitative, et également sous forme de citations extraites des entretiens semi-directifs. Les nouvelles données viennent compléter les résultats de recherches antérieures menées par l'auteur, notamment une enquête auprès d'entrepreneurs moscovites réalisée en 1993 avec l'équipe de recherche de l'Institut d'Economie, et une enquête conduite auprès de 887 chefs de petites entreprises et 210 chefs d'entreprises moyennes ou grandes réalisée en février 1996, à l'occasion du premier Congrès russe des PME et avec le soutien financier de la Chambre russe du Commerce et de l'Industrie ${ }^{14}$. Caractéristiques de l'échantillon L'enquête standardisée a été réalisée entre novembre 1997 et janvier 1998. 227 questionnaires 
complétés ont été recueillis auprès de dirigeants d'entreprises non étatiques dans 21 régions, principalement situées dans la partie européenne de la Russie. Tous les grands secteurs de l'économie sont représentés. La répartition de l'échantillon est la suivante :

Entreprises d'Etat privatisées $18 \%$ Entreprises privées dès leur création $82 \%$ Petites entreprises 79 \% Entreprises grandes et moyennes 21 \% Hommes 75 \% Femmes $25 \%$ Diplômés de l'enseignement supérieur 83 \% Actionnaires de l'entreprise 79 \% Membres d'associations patronales $28 \%$ Entrepreneurs moscovites $19 \%$

Les entretiens approfondis ont été réalisés entre mai 1997 et avril 1998. 96 entretiens ont été enregistrés. Parmi les personnes interrogées, 27 faisaient déjà partie de l'échantillon de l'enquête réalisée en 1993 par le Centre d'Etude des Technologies Politiques. Dans le choix des interviewés, l'accent a été mis principalement sur les secteurs émergents d'activité privée. On trouve dans l'échantillon les dirigeants de plusieurs entreprises prestataires de services financiers.

Bibliographie Andreev N., "Okhrana i 'krycha' : kto obespetchit biznesou jizn' bez strakha » [Sécurité et «krycha » : qui donnera aux entreprises une vie sans crainte ?], Izvestia, 5 février 1998, p. 5. Beauchamp T., Bowie N., "Ethical Theory and Its Application to Business ", in Beauchamp T. \& Bowie N. (dir.). Ethical Theory of Business, Englewood Cliffs (NJ), Prentice-Hall, 1979, p. 14-22. Bhide A., Stevenson H.H., "Why Be Honest If Honesty Doesn't Pay », Harvard Business Review, 68(5), 1990. Chambre russe du Commerce et de l'Industrie, Natsiolnalnaïa programma 'Rossiiskaïa delovaïa koultoura' [Programme national «La culture russe des affaires»], Moscou, 1997. Ermolin V., Kolesnikov A. (1998), « Tchrezvytchainaïa kampania » [Une campagne extraordinaire], Izvestia, 25 novembre, 1998, p. 1. Etzioni A., The Moral Dimension: Toward a New Economics, New York, The Free Press, 1998. Letowska E., Corruption : Towards Greater Transparency? Ethics in the Public Sector: Challenges and Opportunities for OECD Countries, Paris, OECD, 1997. «Moskvitchi vooroujeny do zoubov» [Les moscovites sont armés jusqu'aux dents], Expert, 2000, n 49, p. 9. Polianski A., « Vyche krychi » [Au-dessus du 'toit'], Expert, n², 1996. Radaev V., « Maly biznes i problemy delovoï etiki : nadejdy i realnost' » [Les petites entreprises face aux problèmes d'éthique des affaires : espoirs et réalité], Voprosy Ekonomiki, $\mathrm{n}^{\circ} 7,1996, \mathrm{p}$. 72-82. Radaev V., "Practicing and potential entrepreneurs in Russia », International Journal of Sociology, vol. 27, $\mathrm{n}^{\circ}$ 3, fall 1997, pp. 15-50. Radaev V., "Regional Entrepreneurship : the State of Small Business ", in A Regional Approach to Industrial Restructuring in the Tomsk Region, Russian Federation, Paris, OCDE, 1998, pp.275-319. Radaev V., Formirovanie novykh rossiiskikh rynkov: transaktsionnye izderjki, formy kontrolia i delovaïa etika [La formation des nouveaux marchés russes: coûts de transaction, formes de contrôle et éthique des affaires], Moscou, Centre d'Etude des Technologies Politiques, 1998. Radaev V., «Rossiiskii biznes: strouktoura transaktsionnykh izderjek» [Les affaires en Russie: la structure des coûts de transaction], Obchtchestvennye Naouki i Sovremennost', n 6, 1999, pp. 5-19. Radaev V., The Spread of Violence in Russian Business in the Late 1990s, Program on New Approaches to Russian Security. Policy Memo n 66, Harvard University, août 1999. Radaev V., « Setevoi mir » [Un monde en réseau], Expert, No. 12, 2000, pp. 34-37. Radaev V., «Corruption and Violence in Russian Business in the Late 90's », in Ledeneva, A. \& Kurkchiyan, M. (dir.), Economic Crime in Russia, Londres, Kluwer, 2000. Volkov V., "Violent Entrepreneurship in Post-Communist Russia ", Europe-Asia Studies, n 5 , 
1999. Williamson O.E., The Economic Institutions of Capitalism: Firms, Markets, Relational Contracting, New York, The Free Press, 1985.

\section{NOTES}

1. Traduit de l'anglais par John Crowley.

2. Bhide A., Stevenson H.H., " Why Be Honest If Honesty Doesn't Pay », Harvard Business Review, 68(5), 1990, pp. 121 et 128 ; voir également B Beauchamp T., Bowie N., "Ethical Theory and Its Application to Business ", in Beauchamp T. \& Bowie N. (dir.). Ethical Theory of Business, Englewood Cliffs (NJ), Prentice-Hall, 1979, pp. 14-22 ; Etzioni A., The Moral Dimension : Toward a New Economics, New York, The Free Press, 1998.

3. Pour une présentation plus détaillée des résultats de recherche les plus récents, voir Radaev V., Formirovanie novykh rossiiskikh rynkov : transaktsionnye izderjki, formy kontrolia i delovaïa etika [La formation des nouveaux marchés russes : coûts de transaction, formes de contrôle et éthique des affaires], Moscou, Centre d'Étude des Technologies Politiques, 1998. Voir également l'annexe méthodologique.

4. Chambre russe du Commerce et de l'Industrie. Natsionalnaïa Programma « Rossiiskaïa delovaïa koultoura » (Programme national « La culture russe des affaires »), Moscou, 1997, p. 29.

5. Ibid, p. 178.

6. En Russie, les cours d'arbitrage sont principalement chargées de juger les litiges économiques.

7. Volkov V., « Violent Entrepreneurship in Post-Communist Russia », Europe-Asia Studies, $\mathrm{n}^{\circ}$ 5, 1999, p. 749.

8. Andreev N., « Okhrana i 'krycha' : kto obespetchit biznesou jizn' bez strakha » [Sécurité et 'krycha' : qui donnera aux entreprises une vie sans crainte ?], Izvestia, 5 février 1998, p. 5. Le terme de « krycha » (le toit) désigne la personne ou l'organisation, légale ou criminelle, qui protège les intérêts d'une entreprise ou d'un individu.

9. « Moskvitchi vooroujeny do zoubov » [Les moscovites sont armés jusqu'aux dents], Expert, $2000, \mathrm{n}^{\circ} 49$, p. 9.

10. Letowska E., Corruption : Towards Greater Transparency? Ethics in the Public Sector: Challenges and Opportunities for OECD Countries, Paris, OECD, 1997, pp. 4-5. 11. Polianski A., « Vyche krychi » [Au-dessus du 'toit'], Expert, n² 2, 1996, p. 20.

12. Andreev N., op. cit.

13. Nous voudrions remercier les chercheurs et le personnel du Centre d'Étude des Technologies Politiques (dirigé par I. Bounin), ainsi que V. Goubernatorov (de la Chambre russe du Commerce et de l'Industrie). Sans leurs efforts précieux, ce projet n'aurait pu être conduit.

14. Pour les résultats de ces enquêtes, voir Radaev V., 1996 et 1997 (cf. bibliographie). 
INDEX

Index géographique : Russie

Mots-clés : protection, contrebande et trafic, économie politique, entrepreunariat, mafias et crime organisé 